\title{
Complexity Science as a Frame for Understanding the Management and Delivery of High Quality and Safer Care
}

\author{
Jeffrey Braithwaite (1), Louise A. Ellis (1), \\ Kate Churruca (D), Janet C. Long (i), Peter Hibbert (1), \\ and Robyn Clay-Williams (B)
}

\section{Learning Objectives and Questions Covered in the Chapter}

- How does a linear view of improvement contrast with a complexity science approach?

- The complexity frame makes it harder to manage and deliver high quality and safer caredoes it therefore need to be rejected in favour of simpler improvement models?

- What examples can be brought to bear to show how studies in the complexity frame can lead to good outcomes and positive change?

\subsection{The Complexities of Healthcare}

Over the past two decades, prominent researchers such as Greenhalgh [1], Plsek [2], Leykum [3], Lanham [4], Petticrew [5] and Hawe [6, 7] and their colleagues and teams have promoted using complexity theory to describe and analyse the various dimensions of healthcare organisation [8-12]. Internationally, in parallel, governments have recognised the need to 'think differently'

\footnotetext{
J. Braithwaite $(\bowtie) \cdot$ L. A. Ellis $\cdot$ K. Churruca

J. C. Long · P. Hibbert · R. Clay-Williams

Australian Institute of Health Innovation, Macquarie University, Sydney, NSW, Australia

e-mail: jeffrey.braithwaite@mq.edu.au;

louise.ellis@mq.edu.au; kate.churruca@mq.edu.au; janet.long@mq.edu.au; peter.hibbert@mq.edu.au; robyn.clay-williams@mq.edu.au
}

about healthcare policy and service delivery, but without much traction on how that might be done and what it might mean. Nevertheless, it has now become more common-but by no means universal- to apply a complexity lens to understanding healthcare services and to improving them. This involves greater appreciation of elaborate, intricate, multi-faceted care networks, healthcare ecosystems, layered parts in composite settings, contextual differences across care settings, clinical cultures, multi-agent environments, and the convoluted, challenging, wicked problems [13] these systems throw up. However, with some relatively limited exceptions, the quality and safety fields' interest in complexity has, to date, been largely superficial, both theoretically and empirically [1].

Although it is seen as an emerging field, complexity science is not new; it sprang from knowledge and studies accumulating in disciplines such as sociology, ecology, and evolutionary biology in the 1940s, and with antecedents even earlier in what is broadly called 'systems thinking'. Taking a systems view, healthcare is not just complicated, or layered, or socially dense, or varied, although it is certainly all of these things. Rather, the systems view is based on several fundamental ideas: essentially, that all systems are composed of a set of seemingly discrete but actually interdependent components, defined not just by their inter-relations but by the permeable and shifting boundaries between them. The 
components (people, technology, artefacts, equipment, departments, professions) are combined sometimes in organised and expected ways, and sometimes opportunistically and in unexpected ways. The components are constantly changing, and aggregate such that their collective behaviour is more than the sum of their parts. Complex systems are multidimensional, and characterised by eddying, recurring patterns of behaviour [14]. Complexity has been described as 'a dynamic and constantly emerging set of processes and objects that not only interact with each other, but come to be defined by those interactions' [15].

Healthcare and care delivery systems are powerful examples of a complex adaptive system (CAS) [8, 14]. A healthcare CAS consists of dynamic interactions between different individuals and groups (agents of different types such as bureaucrats, clinicians and patients), as well as their affordances (buildings and artefacts, ranging from stethoscopes to computers to pathology tests to pharmaceuticals). The groups and affordances cohere in a system of relationships which produce roles and behaviours that emerge from those interactions, and which in turn produce outcomes: care, treatment, errors, referrals, discharges and deaths, for example. In essence, CASs are rich in collective behaviour: in healthcare, this means assemblies of loosely or tightly networked doctors, nurses, allied health staff, and scientists; managers of different hues; and policymakers of various kinds and at varying levels, as well as patients and patient groups. They all interact, inter-relating and mediating their behaviours via tools, rules, procedures and equipment, each exercising their skills for common purpose $[2,8]$ - to provide quality care to large numbers of patients.

The interconnections between the agents are dynamic, and the stakeholders interrelate in often surprising ways [16]. This provides challenges to understanding the way systems unfurl over time, to apprehending the system's performance and what drives it, to designing interventions to alter that performance, to improving processes and workflows, and to measuring the outcomes of any interventions in the system $[5,17,18]$. CASs are inherently unpredictable, or it is probably more apt to say that behaviours within them and organisational and clinical outcomes cannot be forecast very far ahead.

\subsection{Managing Complexity}

These complexities of healthcare systems give rise to a range of challenges that clinicians, managers, administrative and support staff, policymakers and researchers face as they carry out their various roles and responsibilities and seek over time to care for, and improve the care for, patients. Yet, the simplest way to manage healthcare is to ignore or deny its complexities. It is easier to imagine a 1:1 correspondence between the intentions for healthcare and the consequential result of those intentions, as seen in Fig. 27.1.

Many people do this: they imagine that the next policy or guideline or mandated change, or quality improvement programme, or procedure, or test result, or new IT system, will be taken up unproblematically on the front lines of care. Contrary to that kind of thinking, there are multiple layers to healthcare complexity [19]; in addition to the dynamics of the care setting (systems complexity), patients come with a range of complex conditions and needs, and diagnosis, treatment and follow-up are rarely unproblematic (medical complexity). As well, patients have intricate life histories, home lives, socioeconomic circumstances, and families, partners, children and parents (situational complexity). All of these complexities co-evolve to evince intractable problems (Fig. 27.2). Indeed, it is at the interstices of these three kinds of complexity depicted in Fig. 27.2 that much wickedness manifests [20]. By wickedness, we mean problems

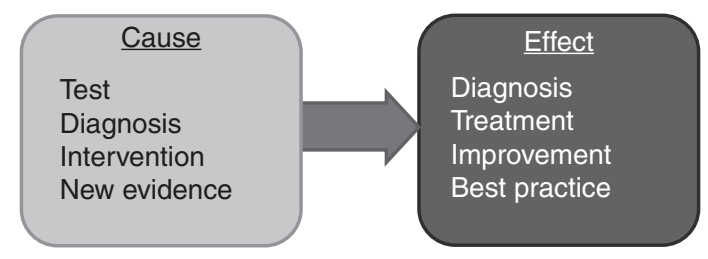

Fig. 27.1 Examples of linear thinking in healthcare. (Authors' conceptualisation) 
Fig. 27.2 Three kinds of complexities that co-evolve to wicked problems. (Reproduced from Kuipers, P., Kendall, E., Ehrlich, C., McIntyre, M., Barber, L., Amsters, D. \& Brownie, S. (2011). Complexity and health care: Health practitioner workforce, services, roles, skills and training to respond to patients with complex needs. Clinical Education and Training Queensland, Queensland Health. ISBN: 9781921707551)

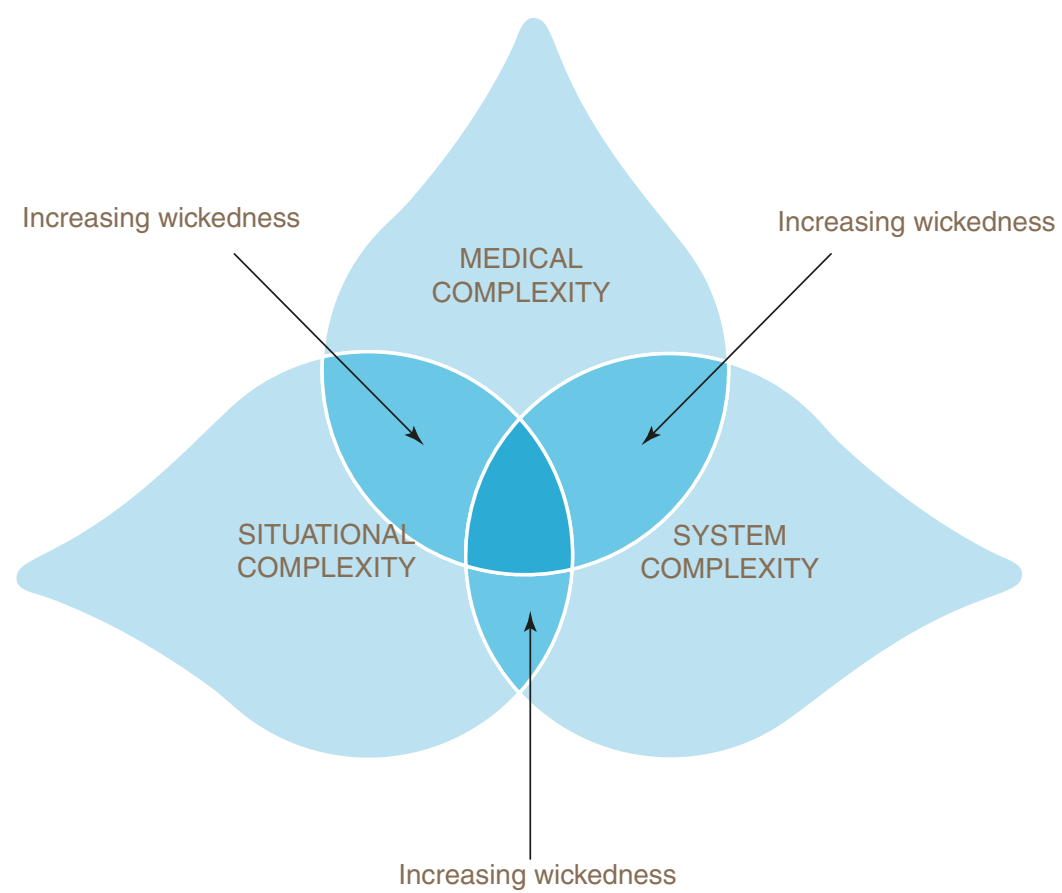

that have considerable degrees of difficulty, are difficult even to define, making them often impossible in principle to solve. How, and the extent to which, any one individual or group (e.g., an executive member or group, or a clinician or clinical team) can effectively manage complexity, or the wicked problems that ensue from that complexity, is not at all clear.

In a complex system, it is not just the different types of complexity that overlap to create challenges, but there are profound uncertainties generated by the inherent complexities [21]. These uncertainties interact (Fig. 27.3). For example, and depicted in Fig. 27.3, uncertainties about the processes of care may be compounded by an unclear diagnosis (labelled in the model scientific uncertainty) and further by a patient's unstable mental state (characterised as personal uncertainty). In many such situations the systems and structures are not understood (denoted as practical uncertainty).

Even more challenging, it is not always possible in healthcare environments for every piece of the jigsaw puzzle to be in place before acting. In fast-paced clinical practice, things happen rapidly, and a prompt response is required. While

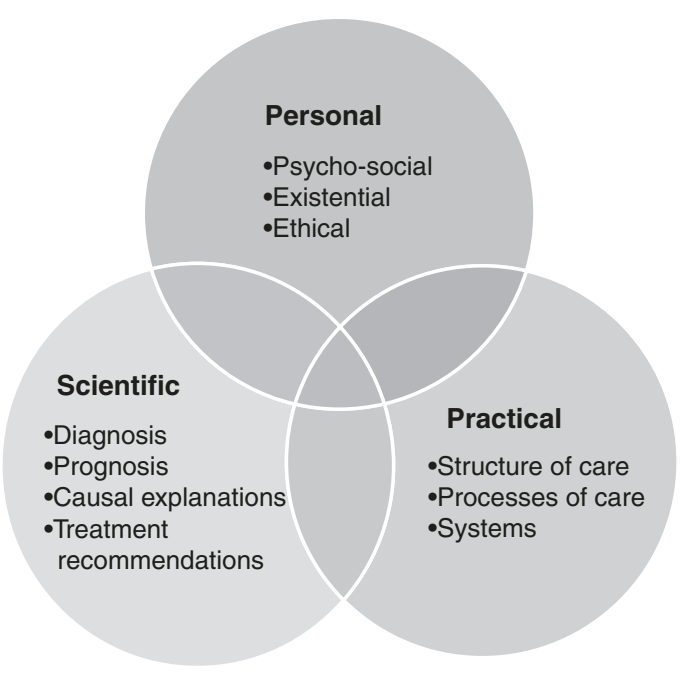

Fig. 27.3 Example of uncertainties interacting in healthcare. (Reproduced from Pomare C, Churruca K, Ellis LA, Long JC \& Braithwaite J. (2019). A revised model of uncertainty in complex healthcare settings: A scoping review. Journal of evaluation in clinical practice, 25(2), 176-182)

clinicians are always under pressure to be exhaustive, they must also be pragmatic, and make room for the next patient. Hollnagel calls this the efficiency-thoroughness trade-off (ETTO) [22]. 
Hence, clinicians must be able to tolerate uncertainty and act even where there is imperfect data and no clearly right path to take. Even when there is thought to be a right path (e.g., a presenting patient with clear symptoms, an apparently accurate diagnosis, and the availability of relevant clinical guidelines stipulating the care to be delivered), the course of the disease can change or the treatment plan can alter, the clinicians may be following their own clinical path (mindlines) rather than the recommended care (guidelines) [23], or the culture within which the clinicians operate might not be conducive to teamwork. And a CAS itself is a dynamic, moving picture, so any treatment plan, or the delivery of care, or the post-treatment follow-up, needs to be updated in the light of the change or new circumstances or information. Even in a stable, well-run organisational ecosystem with a receptive, non-toxic, trustful culture, things can go wrong, variables can alter, the team's composition can change, the carers can come under pressure, or the path to progress can be disrupted and therefore things will not run smoothly.

Another characteristic of complexity in healthcare is the sheer number and variety of stakeholders (e.g., professional groups, clinical specialities, managers, policymakers, regulators) involved in planning for and delivering care. Drawing upon different perspectives and forms of expertise, including the patient's, can potentially facilitate greater understanding of uncertain situations and improve the decision-making process but can also add manifold layers of involvement and interaction [24, 25]. Indeed, in complex systems it is often more appropriate to think about stakeholders and individuals as collective sense-makers [26] rather than rational decisionmakers in the 'if $\mathrm{X}$, then $\mathrm{Y}$ ' mode. This notion focuses on the social aspects of meaning-making: people in CASs spend a considerable amount of energy figuring out what's going on, and what to do next in the light of imperfect information. Indeed, individuals often, even typically, have conflicting understandings of uncertain situations. Sense-making theory says that we must recognise the relative value of the plausibility of a decision or explanation to people over its accu- racy $[12,26]$. For example, healthcare 'huddles', which have been shown to have positive effects on patient safety, leverage complexity principles such as fluidity, adaptiveness, sense-making, trade-offs and meaningful interaction [3, 27]. They provide feasible, credible decisions that the group can accommodate to, live with and action, rather than absolute answers. Clinicians and clinical teams do not make decisions in textbook fashion, or via a structured decision tree, but make sense of the circumstances they find themselves in, and create meaning and take ensuing action emanating from the convergence of information, guidelines, others' perspectives, wishes of patients, and what is viable and possible.

\subsection{Responding to Complexity}

Responding to these inherent complexities of healthcare systems requires letting go of many simplistic explanations and 'one-size-fits-all', or cause-and-effect, solutions. Indeed, in CASs, changes in variables over time are normal, and often appear suddenly, even at times unfathomably or chaotically. Surprise and unpredictability occur frequently: indeed, it has been said that things that have never happened, happen all the time.

Responses to induced or orchestrated or mandated changes can yield unexpected or counterintuitive outcomes. Complex systems, because of their multi-layered nature, exhibit emergence: the properties of the system that arise from interactions at one level down, for example, group behaviour emerges from the relationships and interdependencies of the individuals which make up the group. Healthcare CASs constantly create and re-create such emergent behaviours which by their very nature are unforecastable. This is why the work day people experience as it unfolds is never the same as the one they planned for at the start of the shift.

In addition, in homing in on any part of a CAS, we can discern elements of both selfsimilarity and local nuances. Self-similarity can manifest fractally, at different scales (e.g., features of the culture of the organisation at the team 
level approximate to that of the culture of the department, and then division, and then the whole organisation) or laterally (e.g., one department looks comparable structurally to another). It might seem paradoxical, but healthcare levels or departments, despite being self-similar in some respects, also each operate as unique entities. There are always localised contextual, cultural and structural distinctions. Such local nuances occur as the result of the particular configurations of agents (e.g., nurses, doctors, quality managers, patients) following their internalised rules and shared mental models (e.g., put the patients first, project a good reputation to the outside world, prioritise safety) in that unique setting.

Against such a backdrop, any efforts to introduce a change or quality improvement initiative can be hampered if the complexities of the local context are not taken into account; things don't always work the same in different places and indeed are never adopted as intended, but are always modified by local agents and cultures to suit circumstances [28]. Hence, for change proponents, engagement with agents on a local-level is required and while some aspects of an improvement initiative may be standardised or readily imported from elsewhere, most things will need to be adapted to suit these variable contexts [7]. Even a rigid, highly structured, imposed IT system intended for application universally across a health organisation will be used in vastly different ways by local teams and individuals [29-32].
Responding to complexity also requires letting go of traditional notions of organisational management. CASs manifest in such a way as to thwart attempts at tightly centralised control [33]. No one person can fully comprehend the entire system or claim consistent influence over all agents, for example, something that the issue of localised, nuanced context also underscores. In short, strictly hierarchical management, because it relies on the putative ability of a single person or executive grouping 'at the top' to be in charge, can in reality never cope with all the complexities present in a system such as a hospital or busy community setting, let alone an entire chain, health region or jurisdiction [34]. Alternative models of managing with complexity in mind focus instead on mechanisms or strategies such as distributed leadership, decentralised responsibility, communities of practices, relationshipbuilding, opinion leaders, shared mental models, and networked influence (Fig. 27.4). These hold greater promise in being better able to manage complex environments because information, power and control are spread throughout a CAS, and information that matters is often situated locally $[35,36]$. Control is also distributed, and does not simply reside at the organisational apex. Top-level leaders and managers in complex organisations, then, essentially must focus on broad goals rather than prescriptive instructions-that is, negotiating with colleagues, employees or contractors over what needs to be achieved and then leaving it up to such

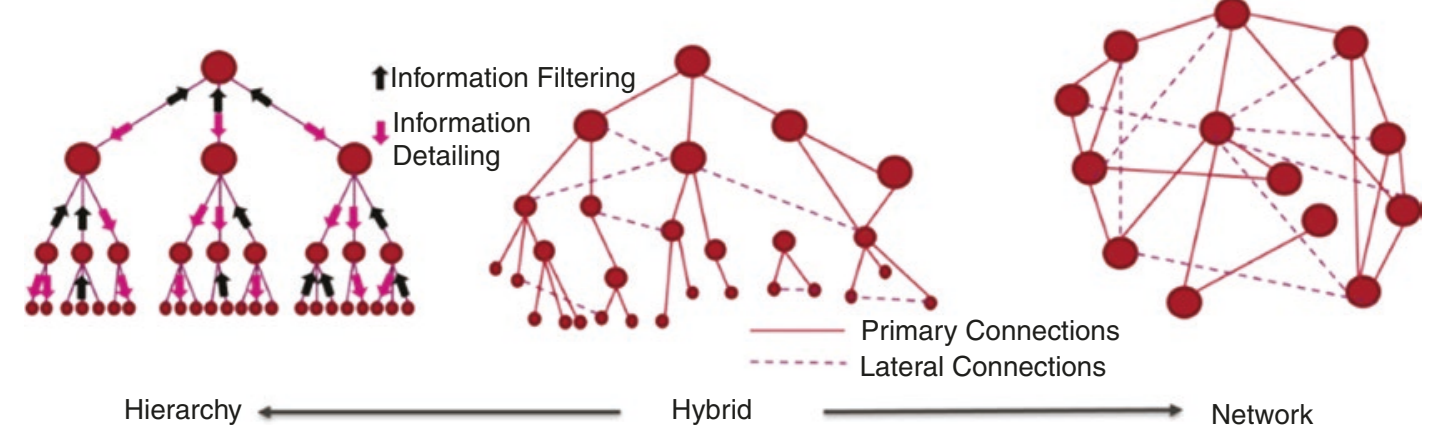

Fig. 27.4 Three types of organisational structures. (Reproduced from Yaneer Bar-Yam (2002) Complexity plexity profile. Encyclopedia of Life Support Systems. Oxford, UK: UNESCO Publishers) 
stakeholders to figure out how they get there [37]. Rather than command and control, ongoing dialogue and feedback between the parties is needed. In CASs, people navigate, manoeuvre and negotiate rather than carry out mandates, prescriptions and detailed requirements. Agency is everywhere; and blind adherence to instructions is in short supply. That is a very considerable dilemma and challenge for managers and leaders in healthcare.

\subsection{Researching Quality and Safety Using Complexity Thinking}

Despite the increasing prominence of complexity, traditional methods for investigating safety, improving quality and implementing risk management in healthcare have typically been based more on linear than complex systems thinking. In linear thinking, unwanted system variability can be controlled and outcomes from an intervention can be generalised, scaled and spread. That logic suggests that the top-down mandates of the apex decision-makers directly, or through causal stages, lead to front-line change. Local problems can be addressed by executives independently of what might be going on in the larger system, and once a problem is solved it is permanently addressed and will no longer need to be monitored. If the intervention is effective, we will see immediate, or almost immediate, results, or at least, responses causally related to or attributed to the top-down decisions, as a chain of events. In linear thinking, we can also apply multiple interventions simultaneously, while assessing them individually.

As we have seen, healthcare is not such a system: it is dynamic, and causality is never fully knowable. Local problems will affect, and be affected by, the larger system. Validity of results will vary with context, and there is a time lagsometimes many years for things that change slowly, such as culture or a new clinical practice-between intervention and results. 'Fix and forget' does not work: we must consider variability, unpredictability and work towards common goals. Planning broadly, but not in detail, for the longer term, and exercising influence, are key methods. Nudge-and-encourage, giving people room to manoeuvre, are more likely to be effective rather than instruction-and-adherence, asking people to comply with organisational requirements.

In such a world, making improvements to care systems, and implementing risk management in healthcare, is more effective when applying multiple methods and respecting the system characteristics rather than searching for one optimal solution. Because systems interact at micro-, meso- and macro-levels, and are configured laterally, across heterarchical structures, we need to capture local and systems-wide information. We also need to understand the interactions between micro-, meso- and macroelements of the CAS, and laterally across the heterarchies. To answer the what?, when? and how much? questions, we can use multilevel statistical modelling. Even more pertinent, we can also model the systems and subsystems, using computational methods such as system dynamics modelling (for aggregated, longitudinal data), social network analysis (for connections and influence), agent-based modelling (for individual or more granular data), and Functional Resonance Analysis Method (FRAM; to understand system variability). To answer the why? and how? questions, we will also need to use more qualitative methods, such as ethnographies, simulations and interviews or focus groups. Integrating multi-method data enables researchers to triangulate. This can help build a rich picture to represent the system we seek to influence, enabling identification of critical issues, key leverage points and potential solutions to improving patient safety or care quality.

\subsection{Real World Examples}

We turn to an analysis of five studies, each of which exemplify our own research, evaluation and interventional efforts in attempting to create safer, better care, and to manage associated risks. Each is a study using complexity science and systems thinking, but in various ways and with differing foci, aims and purposes. We present them at differing levels of granularity, from close 
to the patient, to meso-level studies, to those attempting to influence macro, systems-wide change. The studies are of a group of carers alongside action researchers seeking to improve the way they deliver services to patients with a single clinical condition (our Lynch syndrome study) [38]; an investigation of a coal-face solution to deteriorating patients in acute settings (research on Medical Emergency Teams) [3941]; studies of departmental decision-making, communication and teamwork (social networks in a ward and an emergency department) [42, 43]; an examination of social processes in aggregate, with aligned clinicians, scientists and researchers creating a learning community (Australian Genomics) [44, 45]; and a systemslevel enquiry into patient safety (the Deepening our Understanding of Quality in Australia research programme) [46].

\subsubsection{The Lynch Syndrome Study}

The complexities of work or organisational processes are not always evident at the start of a quality improvement project. An initial or superficial view will end up missing the underlying interdependencies, social processes and emergent behaviours which need to be explored and understood. One way of proceeding is to identify and visualise the focal process as they manifest (work-as-done), rather than as it is believed they manifest (work-as-imagined) [47]. Only then can we design useful interventions. By talking through the steps of how things usually go, and questioning why they are done that way, underlying complexities can be revealed. One of our projects aimed to increase the referral rates of patients with cancer that receive a high-risk result from a screening test $[38,48,49]$. When we initially scoped the project and reviewed the guidelines, it was easy to imagine the system that the clinicians were working in as a relatively simple, linear process of:

1. Screening tests carried out to assess the risk of the cancer being hereditary.

2. Results reported as high or low risk.

3. People with a high-risk result were referred to the genetic service (Fig. 27.5).

It took nine iterations across multiple consultations between the stakeholders involved (surgeons, medical and radiation oncologists, pathologists and genetic specialists) and the research team to develop a process map that was agreed upon as depicting 'referral as done' [38] as seen in Fig. 27.6.

The number of factors influencing whether a high-risk result triggered a referral or not was surprising to all participants in the study. Factors included reluctance to initially overwhelm patients with a 'new' issue ('The results arrive in the first days post-op when the patient is dealing with pain, incontinence and fear of the cancer returning'), failure to remember to refer later, practical issues ('Referral forms are never available', 'There's nowhere in the electronic medical record to record if a referral has been made'), lack of consensus on roles ('It's not my role to refer, just report'), and confusion ('I'm not exactly sure what the report means. Do I refer or not?'). A suite of interventions was developed to effect changes in practices. By mapping the multiplicity of issues arising from the complexity of the context, and presenting them, we allowed

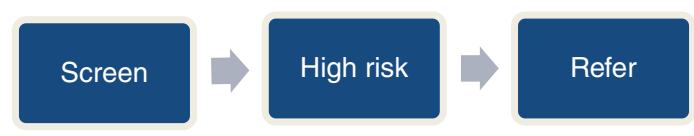

Fig. 27.5 Linear conceptualisation of clinical work amongst Lynch Syndrome clinicians: referral as imagined. (Authors' simplification extracted from Taylor N, Long JC, Debono D, Williams R, Salisbury E, O'Neill S, Eykman E, Braithwaite J \& Chin M. (2016) Achieving behaviour change for detection of Lynch syndrome using the Theoretical Domains Framework Implementation (TDFI) approach: a study protocol. BMC Health Services Research 16:89) 


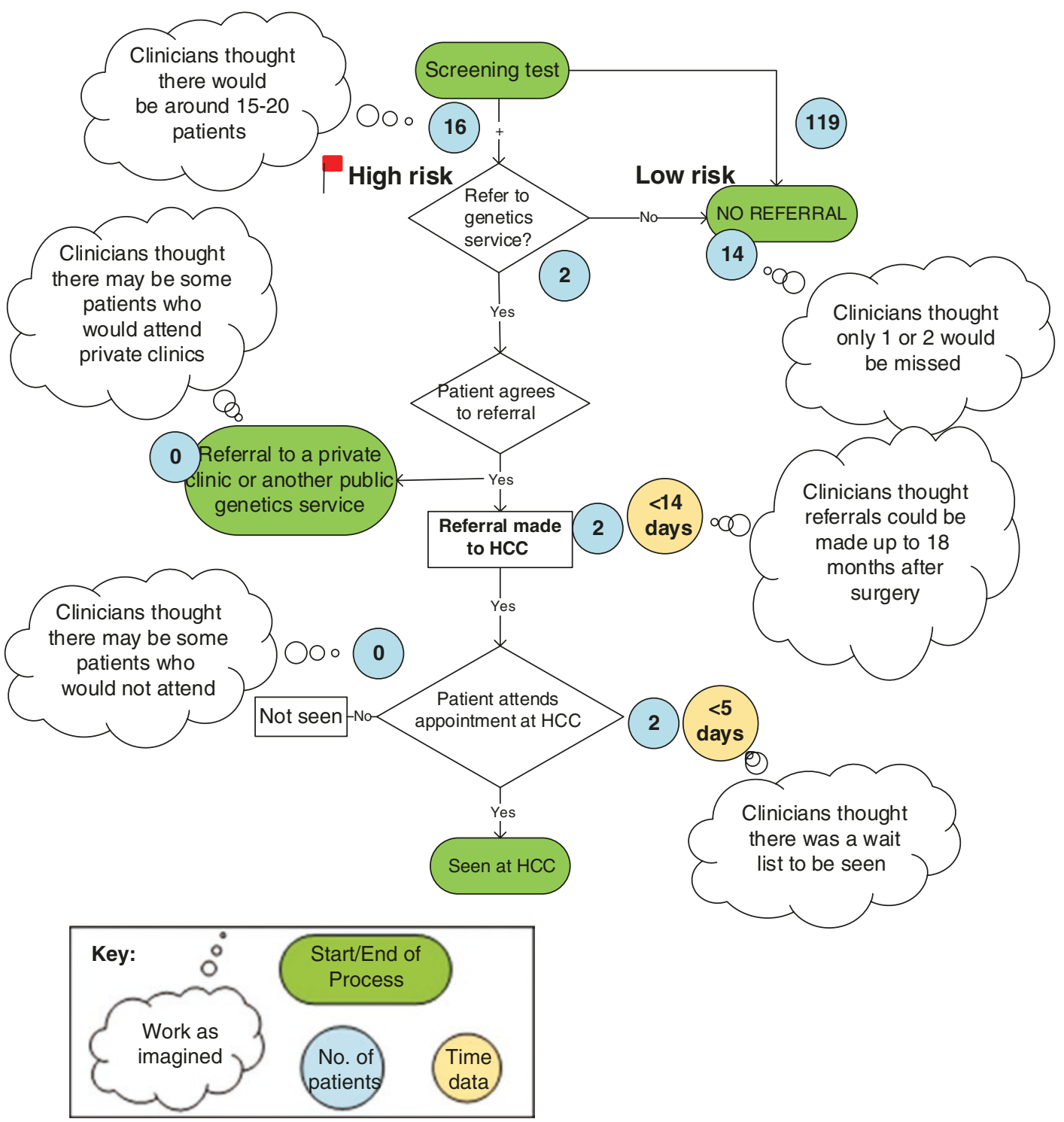

Fig. 27.6 Mapping the referral process for Lynch syndrome. (Authors conceptualisation based on Taylor N, Long JC, Debono D, Williams R, Salisbury E, O’Neill S, Eykman E, Braithwaite J \& Chin M. (2016) Achieving behaviour change for detection of Lynch syndrome using the Theoretical Domains Framework Implementation (TDFI) approach: a study protocol. BMC Health Services Research 16:89) individual clinicians and the team to see processes undertaken in their own and other nearby departments and enabled them to appreciate how their actions could affect up- and down-stream processes. Bringing clinicians from different departments together with the patient as the focus, provided a deeper understanding of other's roles and barriers, helped create a shared mental model, and fostered a whole-of-system approach to the care for patients with this condition. 


\subsubsection{Research on Medical Emergency Teams}

Other features of complexity that confound quality and safety or improvement endeavours are social and cultural influences. The introduction of Medical Emergency Teams in acute settings across the world illustrates this point. When a hospital patient's condition deteriorates, urgent action is required to prevent irreversible harm ('the slippery slope' as seen in Fig. 27.7). Designed by colleagues originally in the USA and Australia, and then involving people internationally, Medical Emergency Teams were developed to provide a rapid response in this situation. If deterioration is detected the Medical Emergency Team can be called in from the intensive care unit to directly manage the patient's decline, the earlier the better.

Two sites where early trials of these teams were conducted had very different outcomes
[28], illuminating the effect of social and cultural norms [50]. The trial was not successful in a well-established hospital in London where the Medical Emergency Teams faced opposition from an entrenched cultural belief that patients were 'owned' by their admitting doctor. This belief clouded both whether the teams were authorised to treat the patient, and where accountability lay for the care of the patient. These issues often caused delays in response which rendered the team's efforts ineffective. The other site was Liverpool Hospital in Sydney, Australia, a newer hospital with an openness to innovation and less opposition to shared accountability for patient outcomes. Here the trial, led by our colleague Ken Hillman, was a success. Today, Medical Emergency Teams are credited with reducing inpatient mortality and cardiac arrests in deteriorating patient cohorts by up to one-third wherever they have been introduced $[39,40,51]$.

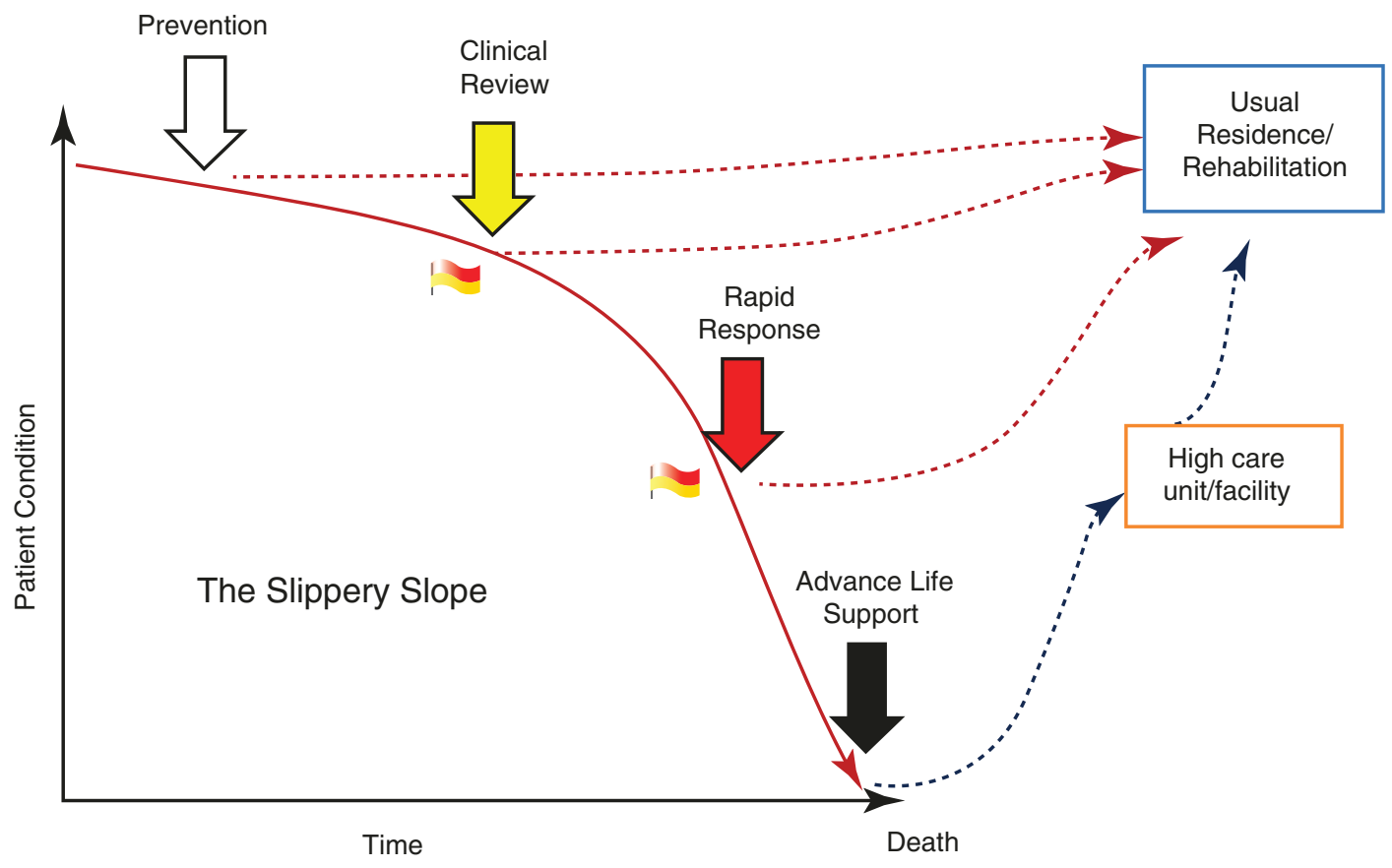

'Between the Flags' intervention on the 'Slippery Slope' of patient deterioration.

Fig. 27.7 Deterioration as a slippery slope in the 'Between the Flags' intervention. (Reproduced from (C) Copyright Clinical Excellence Commission (CEC) 2010) 


\subsubsection{Social Networks in a Ward and an Emergency Department}

Communication and advice-seeking pathways are other key social processes that can affect patient safety and clinical risk but which are often not clearly understood before interventions are commenced [52]. Creswick and colleagues used social network analyses to map these pathways in an Australian Emergency Department, a respiratory and a renal ward $[42,43,53]$, to test assumptions around the nature of communication in the units, who were the key knowledge brokers, and how united their teams were. In one study, the researchers asked staff (Emergency Department $n=109$; respiratory ward $n=47$ ): from whom had they sought advice for medication-related problems recently? They then constructed a sociogram from their replies as seen in Fig. 27.8. They noted that while Emergency Departments are often construed as a single interdisciplinary team, the results show clearly that communication is siloed, with nurses preferring to interact with nurses, doctors with doctors and allied health with allied health. Self-similarity was evident, with the same pattern seen on the ward.

The data facilitated an analysis of key knowledge brokers in the system, including the pharmacist on the ward, and highlighted the need for protecting the roles of those key sources of information. It also revealed the complexities of the 'hidden work' being done by those staff members who were sought out for advice.

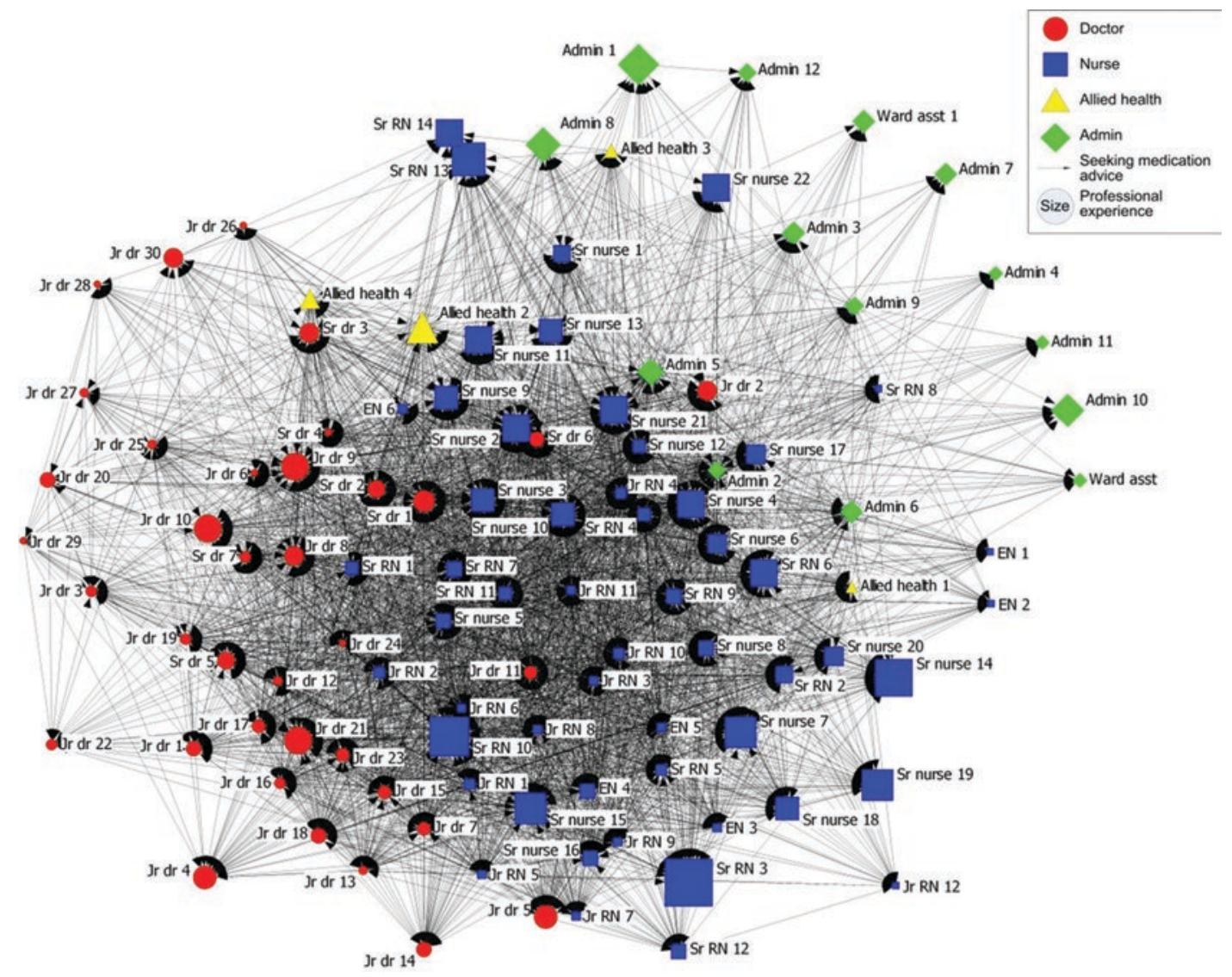

Fig. 27.8 Medication advice-seeking network in an Emergency Department. (Reproduced from: Creswick N, Westbrook JI \& Braithwaite, J. (2009). Understanding communication networks in the emergency department. BMC Health Services Research, 9(1), 247) 


\subsubsection{Australian Genomics as a Learning Community}

Much work has shown how social influences can be potent barriers to interventions and can drive undesirable behaviour [54-56]. They can also be drivers of positive change, of course [38]. But visualising and quantifying social processes is rarely done [44]. Social network analysis is a unique methodology that, as we have seen, maps the interactions between agents in a system and can identify the key players, areas of poor or high connection, and relationship strengths and risks. We used this methodology to examine social influences in the implementation of clinical genomics across Australia [57].

The Australian Genomics Health Alliance (hereafter, Australian Genomics) is a researchfunded, nation-wide collaboration of genomic researchers, practitioners, consumers and operational staff tasked with leading the adoption of clinical genomics into routine healthcare in Australia [58]. Clinical genomics holds great promise for more accurate and rapid diagnosis of rare genetic diseases as well as guiding optimal treatment regimes for people with cancer, producing high quality care. Successful use of clinical genomics relies on interdisciplinary teams of clinical specialists, laboratory scientists, genetic specialists and counsellors to review and interpret the gigabytes of data produced by each genome tested. Early implementation projects with members of Australian Genomics suggested that a potent driver of the successful use of clinical genomics was social influence: learning from one another in the context of actually 'doing genomics'. To map the extent and strength of this influence, we surveyed all members of Australian Genomics $(n=384)$. We asked them: 'What are the strongest influences on the work you are doing towards adoption of clinical genomic practice', and the socially based question: 'Which other members are part of your genomic learning community?'

Results from the 222 respondents confirmed the influence of socially based activities with the most nominated factors being: 'hands on learning' and 'shared decision-making'. 'Formal courses' in contrast did not rate strongly-they were only weakly influential. A sociogram was constructed from respondents' nomination of people in their learning community across Australian Genomics settings (Fig. 27.9).

The largest nodes indicate the people with the most interaction in the network, i.e., the key players. It can be seen that the operational staff are particularly active and that the various groups, while tending towards being clustered, are not insular. Computations of the network show that there is a great deal of mixing across groups. We are using this information to foster more and better learning across groups, to protect key linking and coordinating roles and ultimately to improve care quality.

\subsubsection{The Deepening Our Understanding of Quality in Australia Studies}

The 'Deepening our Understanding of Quality in Australia' (DUQuA) studies represent the culmination of a 5-year multilevel, cross-sectional research programme aiming to identify how quality management systems, clinician leadership and safety culture in Australian hospitals are related to care delivery and patient outcomes [46]. Based on the 'Deepening our Understanding of Quality in Europe' (DUQuE) research in 188 hospitals across seven European countries [59, 60], DUQuA was also able to compare aspects of the Australian and European findings to better understand how quality management is enacted in acute settings. Evidence- or consensus-based measurement tools were designed or modified and then utilised to collect quantitative data on quality management systems at hospital and care pathway levels, department-level clinician safety culture and leadership, clinical treatment processes, patient outcomes, and patient perceptions of safety (Fig. 27.10).

Collection methods included paper-based and electronic surveys, medical record reviews, external audits, and accessing large national datasets. Linear and multilevel modelling were used to identify relationships between quality 


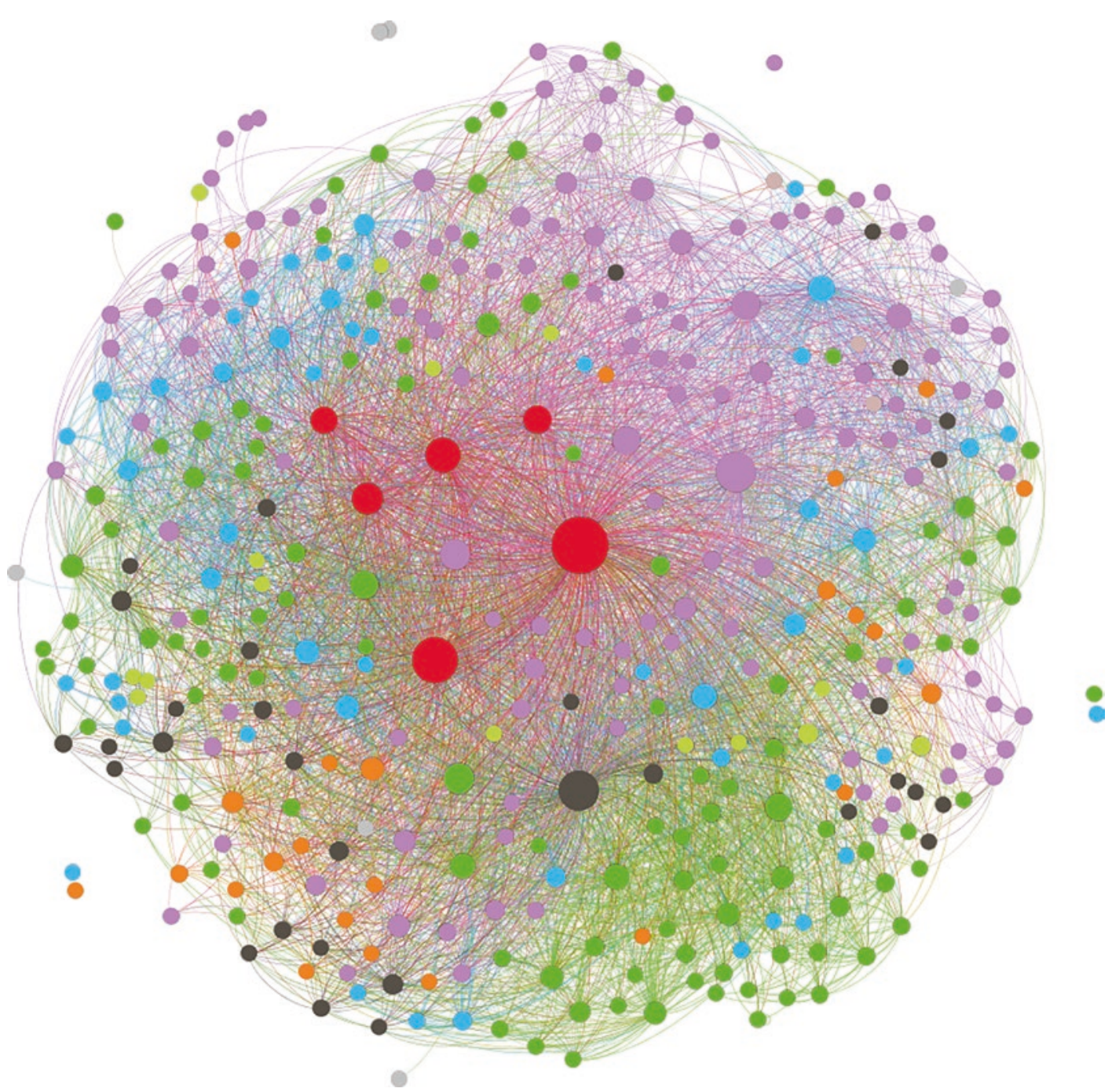

Fig. 27.9 Genomic learning community of members of the Australian Genomics Health Alliance in 2018, where nodes are members and lines are ties. Size of node indi-

management, safety culture and leadership, care delivery and patient outcomes.

DUQuA participants included nearly half $(n=32)$ of the large, acute care public hospitals in Australia. Despite the apparent homogeneity of the group, the variability and complexity of the settings limited our ability to identify strong associations between quality management systems and processes, and patient outcomes. Variation, for example, was often greater within hospitals than between them. In some instances, such as implementation of evidence-based patient safety measures, Australia's mandatory accredi- cates importance in the network. Colours represent the various working groups $(n=22)$; red indicates national operational staff. (Created with Gephi 0.9)

tation programme involving assessment of performance against ten National Standards [61], meant that there was little variability between comparative hospital safety performance at the organisation level. At the care pathway level, however, for the acute myocardial infarction, stroke and hip fracture conditions that were assessed, there was significant variation in leadership and safety culture between conditions within the same hospital.

At individual hospitals, DUQuA has enabled us, through statistical modelling, to answer the what?, when? and how much? questions about 


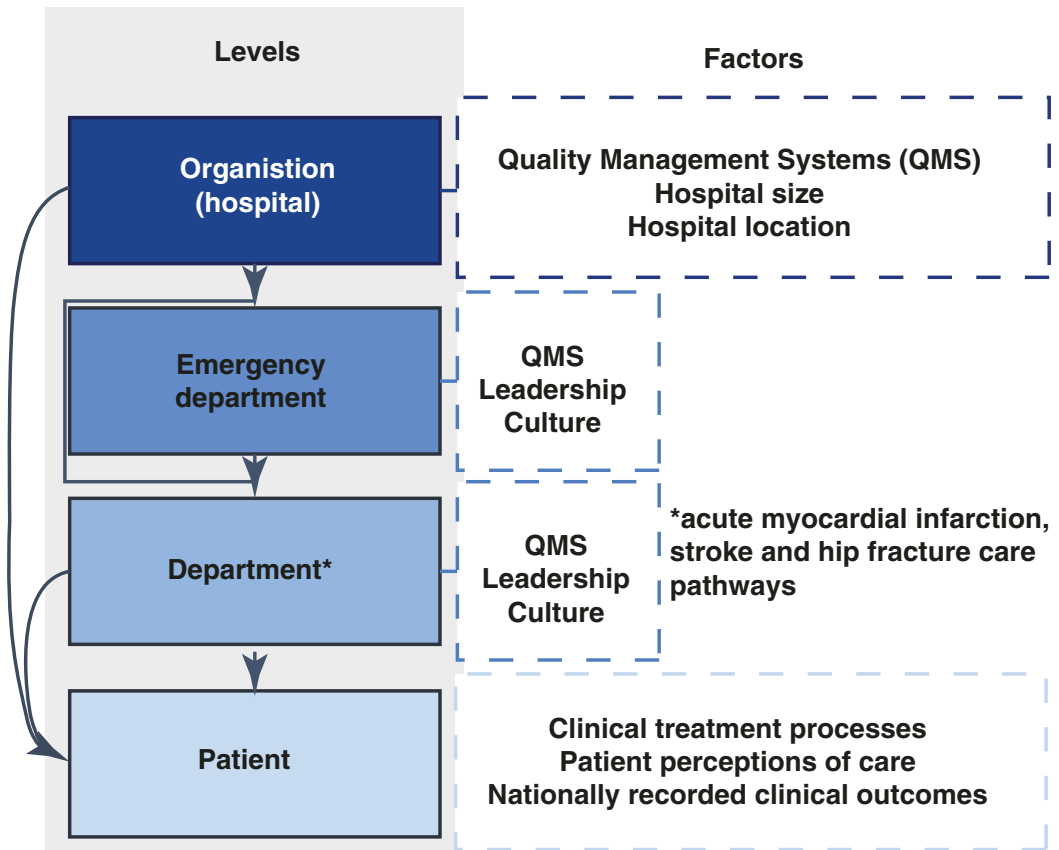

Fig. 27.10 Levels and factors investigated in the Deepening our Understanding of Quality in Healthcare in Australia study. (Reproduced from Braithwaite J, ClayWilliams R, Taylor N, Ting HP, Winata T, Hogden E, Li Z, Selwood A, Warwick M, Hibbert P, Arnolda G. Deepening

our acute care hospitals, and pointed to where we might best concentrate our efforts for future work. It has also enabled us to provide customised benchmarking data and links to evidence-based ideas for improvement for each hospital in the form of a report, alongside validated measurement tools to aid hospital executives and clinical leaders in implementing future quality improvement activities, and measuring their efficacy.

\subsection{Extending These Ideas and Studies to the Future Organisation of Quality and Safety}

We have made the case that complexity science is a gateway to understanding healthcare organisations and the quality and safety of the care they provide. Complexity science aids understanding of the intricate, multi-agent, interactive system under consideration. As we have seen, thinking of healthcare settings as CASs rather than linear our Understanding of Quality in Australia (DUQuA): an overview of a nation-wide, multilevel analysis of relationships between quality management systems and patient factors in 32 hospitals. International Journal for Quality in Health Care. 2020;32(S1):8-2.)

systems facilitates a deeper appreciation of careas-delivered rather than as-imagined-to-be-delivered, and adds texture and richness to the understanding of what needs to be tackled in the pursuit of safer, higher quality, better care.

The alternative, a more linear depiction of the system within which care takes place, misses much and assumes or portrays that the task of improvement is more manageable than it is. Complexity thinking does not make the problems we are trying to solve in intervening to create safer and higher quality care any easier. To the contrary, it is in reality a frontal attack on simplistic thinking, and those who assume the readiness of systems to accommodate new ideas, techniques, programmes and technology and who believe that take-up, scale and spread inevitably flow from decisions made at the top of the organisation chart or in response to research findings. Traditional thinking also assumes there is sufficient absorptive capacity: the system can do what is being asked of it when safer or higher quality care is demanded of it. In linear 
representations the essential logic is chain reasoning, i.e., one thing leads to another in a cascading sequence. That change model at its most basic is 'do this' in a prescribed way, and when things don't work out 'do more' or 'try harder'. In complexity representations, the essential logic is different. Complexity thinking is predicated on understanding multi-stakeholder, interacting relationships, giving consideration to the fact that the whole is greater than, or at the very least different from, the sum of the parts. That change model is based on non-linearity: the system is governed by feedback and is constantly adapting, with emergent behaviours and, due to the sheer amount of interactions and interdependencies, is inherently unpredictable. What seems on the surface as a caused result (attributed to the actions of a decision-maker, or the executive team), can be coupled, and related to that decision and the accompanying actions, but can also be unlinkeda mirage correlation, or an independent event, or an outcome of multiple interactions, or networked influence [62]. What masquerades in a CAS as causality, in summary, is sometimes, even mostly, not.

As we have seen, complexity science led us to ask, of Lynch syndrome-type studies, how does clinical work actually unfold, in contradistinction to how people think it does? We considered, of Medical Emergency Team-type interventions: how long does spread take, even of a relatively easy-to-apprehend idea, but whose time has come, and what cultural factors enabled or impeded the emergence of Medical Emergency Teams, and their subsequent take-up? A complexity science lens was also mobilised to point to how networked behaviours were mapped to understand the connections on the front line of care (in Emergency Departments and wards) and social processes in learning communities across a country (in Australian Genomics). And, in our final example, it helped us to home in on the care actually provided in multiple large hospitals, uncovering which variables are important (but not deterministic) in quality improvement, systems-wide (the DUQuA research programme).

\subsection{Where to From Here?}

Encouraging people to adopt a complexity frame of reference, and doing research or designing interventions using such an approach, where strict generalisability, direct causation, and predictability are ruled out, can be daunting. It is easier to maintain a rationalisation that says, essentially: let's design straight-line interventions, implement such projects as intended, and prescribe the solution to the front lines in order for them to take up the evidence directly. This involves standardised procedures, mandated programmes, and pre-defined, structured tools or techniques. Once implemented, so this instrumental logic goes, we can observe the improvement that will inevitably and unproblematically follow as a result of these initiatives.

But in complex systems, things are not readily decomposable into parts. CASs are organic, not mechanistic; frogs, not bicycles [63].

We believe linear models have limited applications in CASs generally, and healthcare specifically. The job of scholars, improvement agents, quality and safety specialists and policymakers is not to ice-skate elegantly over the top of problems or to construe them simply, but to grapple with them in all their gritty reality. In non-linear, complex systems, end-point changes can be disproportional to the inputs. Newton's clockwork universe of logical, machine-like health systems that change in direct response to the requirements placed on them can only take us - and indeed has only taken us-so far. Quantum mechanics, with its inherent complexity, unpredictability and uncertainty principle is a much more apt metaphor for change. As more people realise this, they will be more likely to appreciate the multidimensional task of improving care quality and making things safer for patients. This way leads to tools such as FRAM [64], social network analyses [57] and system dynamics modelling. It paves the way to an understanding of the resilient properties of health systems [47]. It tells us to search for a deeper appreciation for the characteristics of the system, e.g., its absorptive capacity, contextual 
richness and nuanced cultures. It invited us to consider the characteristics of the agents and stakeholder groups who manoeuvre, negotiate, trade-off and navigate their settings. These are the features of healthcare that anyone interested in researching, leading or delivering high quality care, or managing clinical risk, must factor into their thinking and practices.

\subsection{Recommendations}

1. Sensitise those with responsibility for leading, managing, improving or researching care settings to a systems view.

2. Train sufficient staff in the tools of complexity: FRAM, network analyses, system dynamics modelling, process mapping, and the like.

3. Approach quality and safety and risk management activities with a knowledge of complexity science, sense-making, and non-linearity rather than as a set of linear problems amenable to simplistic causal change logic.

4. Consider how our studies, borrowing from complexity theory, have resisted simplifying the challenges, but have nevertheless made progress in understanding care systems and their improvement.

\section{References}

1. Greenhalgh T, Papoutsi C. Studying complexity in health services research: desperately seeking an overdue paradigm shift. BMC Med. 2018;16:95.

2. Plsek PE, Greenhalgh T. The challenge of complexity in health care. BMJ. 2001;323:625.

3. Provost SM, Lanham HJ, Leykum LK, McDaniel RR Jr, Pugh J. Health care huddles: managing complexity to achieve high reliability. Health Care Manage Rev. 2015;40:2-12.

4. Lanham HJ, Leykum LK, Taylor BS, McCannon CJ, Lindberg C, Lester RT. How complexity science can inform scale-up and spread in health care: understanding the role of self-organization in variation across local contexts. Soc Sci Med. 2013;93:194-202.

5. Petticrew M. When are complex interventions 'complex'? When are simple interventions 'simple'? Eur J Pub Health. 2011;21:397-8.
6. Hawe P. Lessons from complex interventions to improve health. Annu Rev Public Health. 2015;36:307-23.

7. Hawe P, Shiell A, Riley T. Complex interventions: how "out of control" can a randomised controlled trial be? BMJ. 2004;328:1561.

8. Braithwaite J, Clay-Williams R, Nugus P, Plumb J. Health care as a complex adaptive system. In: Hollnagel E, Braithwaite J, Wears RL, editors. Resilient health care. Surrey, UK: Ashgate Publishing; 2013. p. 57-73.

9. Anderson RA, Issel LM, McDaniel RR Jr. Nursing homes as complex adaptive systems: relationship between management practice and resident outcomes. Nurs Res. 2003;52:12-21.

10. Anderson RA, Corazzini KN, McDaniel RR Jr. Complexity science and the dynamics of climate and communication: reducing nursing home turnover. Gerontologist. 2004;44:378-88.

11. Miller WL, McDaniel RR, Crabtree BF, Stange KC. Practice jazz: understanding variation in family practices using complexity science. J Fam Pract. 2001;50:872-8.

12. McDaniel RR, Dean JD. Complexity science and health care management. In: Friedman LH, Goes J, Savage GT, editors. Advances in health care management, vol. 2. Bingley, UK: Emerald Group Publishing Limited; 2001. p. 11-36.

13. Cunningham FC, Ranmuthugala G, Westbrook JI, Braithwaite J. Tackling the wicked problem of health networks: the design of an evaluation framework. BMJ Open. 2019;9:e024231.

14. Braithwaite J, Churruca K, Ellis LA, Long JC, ClayWilliams R, Damen N, Herkes J, Pomare C, Ludlow $\mathrm{K}$. Complexity science in healthcare - aspirations, approaches, applications and accomplishments: a white paper. Sydney: Australian Institute of Health Innovation, Macquarie University; 2017.

15. Cohn S, Clinch M, Bunn C, Stronge P. Entangled complexity: why complex interventions are just not complicated enough. J Health Serv Res Policy. 2013;18:40-3.

16. Rychetnik L, Frommer M, Hawe P, Shiell A. Criteria for evaluating evidence on public health interventions. J Epidemiol Commun Health. 2002;56:119.

17. Craig P, Dieppe P, Macintyre S, Michie S, Nazareth I, Petticrew M. Developing and evaluating complex interventions: the new Medical Research Council guidance. BMJ. 2008;337:a1655.

18. Datta J, Petticrew M. Challenges to evaluating complex interventions: a content analysis of published papers. BMC Public Health. 2013;13:568.

19. Braithwaite J, Churruca K, Ellis LA. Can we fix the uber-complexities of healthcare? J R Soc Med. 2017;110:392-4.

20. Rittel HWJ, Webber MM. Dilemmas in a general theory of planning. Policy Sci. 1973;4:155-69. 
21. Pomare C, Churruca K, Ellis LA, Long JC, Braithwaite J. A revised model of uncertainty in complex healthcare settings: a scoping review. J Eval Clin Pract. 2019;25:176-82.

22. Hollnagel E. The ETTO principle: efficiencythoroughness trade-off. Boca Raton, FL: Routledge; 2018.

23. Gabbay J, Al M. Evidence based guidelines or collectively constructed "mindlines?" Ethnographic study of knowledge management in primary care. BMJ. 2004;329:1013.

24. Politi MC, Street RL. The importance of communication in collaborative decision making: facilitating shared mind and the management of uncertainty. J Eval Clin Pract. 2011;17:579-84.

25. Pomare C, Long JC, Ellis LA, Churruca K, Braithwaite J. Interprofessional collaboration in mental health settings: a social network analysis. J Interprof Care. 2019;33:497-503.

26. Weick KE. Sensemaking in organizations. Thousand Oaks, CA: SAGE Publication; 1995.

27. Jordan ME, Lanham HJ, Crabtree BF, Nutting PA, Miller WL, Stange KC, McDaniel RR Jr. The role of conversation in health care interventions: enabling sensemaking and learning. Implement Sci. 2009;4:15.

28. Braithwaite J, Churruca K, Long JC, Ellis LA, Herkes J. When complexity science meets implementation science: a theoretical and empirical analysis of systems change. BMC Med. 2018;16:63.

29. Debono D, Braithwaite J. Workarounds in nursing practice in acute care: a case of a health care arms race? In: Wears RL, Hollnagel E, Braithwaite J, editors. Resilient health care, The resilience of everyday clinical work, vol. 2. Surrey, UK: Ashgate Publishing; 2015.

30. Westbrook JI, Duffield C, Li L, Creswick NJ. How much time do nurses have for patients? A longitudinal study quantifying hospital nurses' patterns of task time distribution and interactions with health professionals. BMC Health Serv Res. 2011;11:319.

31. Greenhalgh T, Wherton J, Papoutsi C, Lynch J, Hughes G, A'Court C, Hinder S, Fahy N, Proctor R, Shaw S. Beyond adoption: a new framework for theorizing and evaluating nonadoption, abandonment, and challenges to the scale-up, spread, and sustainability of health and care technologies. J Med Internet Res. 2017; 19:e367.

32. Greenhalgh T, Wherton J, Papoutsi C, Lynch J, Hughes G, A'Court C, Hinder S, Proctor R, Shaw $\mathrm{S}$. Analysing the role of complexity in explaining the fortunes of technology programmes: empirical application of the NASSS framework. BMC Med. 2018;16:66.

33. Greenhalgh T, Plsek P, Wilson T, Fraser S, Holt T. Response to 'The appropriation of complexity theory in health care'. J Health Serv Res Policy. 2010;15:115-7.

34. Bar-Yam Y. Complexity rising: from human beings to human civilization, a complexity profile. In:
UNESCO, editor. Encyclopedia of life support systems. Oxford, UK: UNESCO Publishers; 2002.

35. Braithwaite J, Runciman WB, Merry AF. Towards safer, better healthcare: harnessing the natural properties of complex sociotechnical systems. Qual Saf Health Care. 2009;18:37.

36. Greenfield D, Braithwaite J, Pawsey M, Johnson B, Robinson M. Distributed leadership to mobilise capacity for accreditation research. J Health Organ Manag. 2009;23:255-67.

37. Wilson T, Holt T, Greenhalgh T. Complexity and clinical care. BMJ. 2001;323:685.

38. Long JC, Debono D, Williams R, Salisbury E, O'Neill $\mathrm{S}$, Eykman E, Butler J, Rawson R, Phan-Thien K-C, Thompson SR, Braithwaite J, Chin M, Taylor $\mathrm{N}$. Using behaviour change and implementation science to address low referral rates in oncology. BMC Health Serv Res. 2018;18:904.

39. Chan PS, Jain R, Nallmothu BK, Berg RA, Sasson C. Rapid response teams: a systematic review and meta-analysis. JAMA Intern Med. 2010;170:18-26.

40. Chen J, Bellomo R, Flabouris A, Hillman K, Finfer $\mathrm{S}$, The MERIT Study Investigators for the Simpson Centre, the ANZICS Clinical Trials Group. The relationship between early emergency team calls and serious adverse events. Crit Care Med. 2009;37:148-53.

41. Hughes C, Pain C, Braithwaite J, Hillman K. 'Between the flags': implementing a rapid response system at scale. BMJ Qual Saf. 2014;23:714.

42. Creswick N, Westbrook JI. Social network analysis of medication advice-seeking interactions among staff in an Australian hospital. Int J Med Inform. 2010;79:e116-e25.

43. Creswick N, Westbrook JI, Braithwaite J. Understanding communication networks in the Emergency Department. BMC Health Serv Res. 2009;9:247.

44. Long JC, Cunningham FC, Carswell P, Braithwaite J. Who are the key players in a new translational research network? BMC Health Serv Res. 2013;13:338.

45. Long JC, Pomare C, Best S, Boughtwood T, North K, Ellis LA, Churruca K, Braithwaite J. Building a learning community of Australian clinical genomics: a social network study of the Australian Genomic Health Alliance. BMC Med. 2019;17:44.

46. Braithwaite J, Clay-Williams R, Taylor N, Ting HP, Winata T, Arnolda G, Sunol R, Grone O, Wagner C, Klazinga NS, Donaldson L, Dowton SB. Bending the quality curve. Int J Qual Health Care. 2020;32(Suppl_1):1-7.

47. Braithwaite J, Wears RL, Hollnagel E, editors. Resilient health care, Reconciling work-as-imagined and work-as-done, vol. 3. Abingdon, UK: Taylor \& Francis; 2017.

48. Long JC, Winata T, Debono D, Phan-Tien K-C, Zhu C, Taylor N. Process evaluation of a behaviour change approach to improving clinical practice for detecting hereditary cancer. BMC Health Serv Res. 2019;19:180. 
49. Taylor N, Long JC, Debono D, Williams R, Salisbury E, O’Neill S, Eykman E, Braithwaite J, Chin M. Achieving behaviour change for detection of Lynch syndrome using the Theoretical Domains Framework Implementation (TDFI) approach: a study protocol. BMC Health Serv Res. 2016;16:89.

50. Braithwaite J, Clay-Williams R, Vecellio E, Marks D, Hooper T, Westbrook M, Westbrook J, Blakely B, Ludlow K. The basis of clinical tribalism, hierarchy and stereotyping: a laboratory-controlled teamwork experiment. BMJ Open. 2016;6:e012467.

51. Chen J, Ou L, Flabouris A, Hillman K, Bellomo R, Parr M. Impact of a standardized rapid response system on outcomes in a large healthcare jurisdiction. Resuscitation. 2016;107:47-56.

52. The Joint Commission-Office of Quality and Patient Safety. Sentinel event data - root causes by event type 2004-2015. Oak Brook, IL: The Joint CommissionOffice of Quality and Patient Safety; 2016.

53. Creswick N, Westbrook JI. Who do hospital physicians and nurses go to for advice about medications? A social network analysis and examination of prescribing error rates. J Patient Saf. 2015;11:152-9.

54. Lipworth W, Taylor N, Braithwaite J. Can the theoretical domains framework account for the implementation of clinical quality interventions? BMC Health Serv Res. 2013;13:530.

55. Kelly MP, Barker M. Why is changing healthrelated behaviour so difficult? Public Health. 2016;136:109-16.

56. Damschroder LJ, Aron DC, Keith RE, Kirsh SR, Alexander JA, Lowery JC. Fostering implementation of health services research findings into practice: a consolidated framework for advancing implementation science. Implement Sci. 2009;4:50.
57. Long JC, Hibbert P, Braithwaite J. Structuring successful collaboration: a longitudinal social network analysis of a translational research network. Implement Sci. 2016;11:19.

58. Australian Genomic Health Alliance. 2019. https:// wwwaustraliangenomicsorgau/. Accessed 18 Oct 2019.

59. Wagner C, Groene O, Thompson CA, Dersarkissian M, Klazinga NS, Arah OA, Sunol R, et al. DUQuE quality management measures: associations between quality management at hospital and pathway levels. Int J Qual Health Care. 2014;26:66-73.

60. Groene O, Klazinga N, Wagner C, Arah OA, Thompson A, Bruneau C, Sunol R, on behalf of the DUQuE Research Project. Investigating organizational quality improvement systems, patient empowerment, organizational culture, professional involvement and the quality of care in European hospitals: the 'Deepening our Understanding of Quality Improvement in Europe (DUQuE)' project. BMC Health Serv Res. 2010;10:281.

61. Australian Commission on Safety and Quality in Health Care. National safety and quality health service standards. Sydney: Australian Commission on Safety and Quality in Health Care; 2012.

62. Sugihara G, May R, Ye H, Hsieh C-H, Deyle E, Fogarty M, Munch S. Detecting causality in complex ecosystems. Science. 2012;338:496.

63. Mant A. Intelligent leadership. 2nd ed. Sydney: Allen \& Unwin; 2000.

64. Hollnagel E. FRAM: The Functional Resonance Analysis Method: modelling complex socio-technical systems. Boca Raton, FL: CRC Press; 2012.

Open Access This chapter is licensed under the terms of the Creative Commons Attribution 4.0 International License (http://creativecommons.org/licenses/by/4.0/), which permits use, sharing, adaptation, distribution and reproduction in any medium or format, as long as you give appropriate credit to the original author(s) and the source, provide a link to the Creative Commons license and indicate if changes were made.

The images or other third party material in this chapter are included in the chapter's Creative Commons license, unless indicated otherwise in a credit line to the material. If material is not included in the chapter's Creative Commons license and your intended use is not permitted by statutory regulation or exceeds the permitted use, you will need to obtain permission directly from the copyright holder.

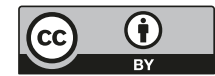

